[CONTRIBUTION FROM THY FIXED NITROGEN RESEARCH LABORATORY. ${ }^{2}$ ]

\title{
THE ENTROPY OF ELECTRON GAS.
}

\author{
By Richard C. Tolman. \\ Received April 21, 1921.
}

\section{Introduction.}

It has become increasingly evident that physical chemists can achieve considerable simplification in mode of expression and thought by treating electrons as the atoms of a chemical substance. Just as we speak of the substance chloride ion and write for it the formula $\mathrm{Cl}^{-}$, we may speak of the substance electron and write for it the formula $\mathrm{E}^{-}$, and the use of such symbols in electrochemistry has already become usual.

In the case of the electrons given off from a heated filament we may properly speak of the substance electron gas. Richardson ${ }^{2}$ and his coworkers have indeed shown that these electrons are actually emitted with velocities which are distributed in the different directions in accordance with Maxwell's distribution law for the molecules of a gas which cross any plane boundary. Whatever the distribution of emitting velocities may be, however, if we had an evacuated vessel with walls made from the filament substance and raised the walls to a high temperature, the vessel would fill with electron gas which would exert a definite vapor pressure, depending on the temperature of the walls. Laue ${ }^{3}$ has recently made an elaborate investigation of the conditions under which this gas will obey the perfect gas laws, while the application of thermodynamic reasoning to the behavior of the gas has been made familiar by the work of $\mathrm{H}$. A. Wilson, ${ }^{4}$ Richardson ${ }^{2}$ and others.

Owing to the very considerable force of repulsion between electrons, the conditions under which this gas will not deviate appreciably in behavior from a perfect monatomic gas are more stringent than for most monatomic substances, which usually have a moderate attraction between molecules rather than a strong repulsion. In order for electron gas to obey the perfect gas laws, the concentration must be low enough and the temperature high enough so that the effects produced by the repulsive force between the electrons can be neglected in comparison with effects produced by their thermal agitation. Furthermore, on account of the mutual repulsion of the electrons, the dimensions of the containing vessel must be small unless we are willing to allow a higher concentration at the periphery than in the center of the gas. And finally the containing walls

1 Published by permission of the Chief of Ordnance.

${ }^{2}$ Richardson, "The Emission of Electricity from Hot Bodies," Longmans, Green and Co., London, 1916.

' Laue, Ann. Physik, 58, 695 (1919).

' H. A. Wilson, Phil. Trans., 202A, 258 (1903). 
must all be of the same material and at the same temperature since otherwise the Volta effect will set up differences in electric potential between different portions of the vessel which will destroy the uniform concentration of the gas.

It should be noted that these restrictions which are necessary in order that the perfect gas laws may apply are of the same nature and merely more stringent than those encountered in the case of more familiar gases. All gases obey the perfect gas laws more closely as their concentration is made lower and their temperature higher. Furthermore in large masses of gas the gravitational attraction of the gas for itself leads to an increased concentration at the center analogous to the increased concentration at the periphery in the case of electron gas. Finally the local disturbances in concentration which would be produced by differences in potential between different parts of the containing walls are entirely analagous to the differences in concentration which may be produced in ordinary gases by the gravitational effect of external bodies.

Perhaps the most distinguishing characteristic of electron gas is that it is apparently a gas which is plus que parfait at all temperatures and pressures while most gases are moins que parfait throughout the greater range of temperature and pressure. It should also be pointed out that the mechanism by which electron gas exerts its pressure is obviously not that of the older kinetic theories which assumed a bombardment of the walls by rigid elastic molecules. This consideration, however, need cause us no concern, since we have already learned even in the case of more familiar gases to regard the above mechanism at least as no better than a first approximation. In the case of any gas dilute enough the exact nature of the mechanism by which the momentum is transferred to the walls has no effect on the final value of the pressure.

\section{Theoretical Equation for the Entropy of Electron Gas.}

Assuming now that we have electron gas under such conditions that it obeys the laws for a perfect monatomic gas law we may evidently write for its entropy per mol,

$$
S=5 / 2 R \ln T-R \ln p+\text { const. }
$$

With regard to the constant occurring in Equation 1, Laue ${ }^{5}$ has already made the suggestion that this constant will depend on molecular weight in the same way as has been found for other monatomic gases. If this be true, we may rewrite Equation 1 in the form

$$
S=5 / 2 R \ln T-R \ln p+8 / 2 R \ln M+S_{1}
$$

where $M$ is the molecular weight of the electron and $S_{1}$ has the same value for all monatomic gases

s Laue, Jahr. Radioakt. Elektronik, 15, 257 (1918). 
In a recent article ${ }^{6}$ the anthor has shown the possibility of deriving Equation 2 in a very simple way from the principle of similitude or relativity of size, and has found that the entropy of a number of monatomic gases at $25^{\circ}$ is closely given by Equation 2 when $S_{1}$ is given the value $24.27,7 \mathrm{~S}$ and $R$ being in calories per degree, $T$ in degrees centigrade absolute, $p$ in dynes per sq. cm. and $M$ the molecular weight in grams.

The main purpose of the present article is to show that the entropy of electron gas as calculated from Equation 2 actually agrees with the entropy of the electron gas in equilibrium with tungsten, tantalum and molybdenum filaments at $2000^{\circ}$ absolute as calculated from the heat known to be absorbed when electrons are emitted from the hot filaments. Having thus assured ourselves of the probable validity of Equation 2, we shall then make use of this equation in a following article to study the thermal ionization of gaseous vapors a.t high temperatures.

Pressure of Electron Gas in Equilibrium with Hot Filaments.In order to apply Equation 2 to the electron gas in equilibrium with hot filaments, we must evidently have a value for the pressure of the gas in equilibrium with the filament. Now there is a well-known relation between the pressure of a gas $p$, and the number of mols of gas $N$ which cross a plane surface of unit area in unit time. This relation may be written in the form,

$$
N=\frac{p}{\sqrt{2 \pi M R T}}
$$

and can be simply derived merely from the assumption that the velocities of the molecules are distributed in accordance with Maxwell's distribution law.

In the case of a filament in equilibrium with an electron gas, Equation 3 will determine the number of electrons impinging on unit area of the filament in unit time. Since we have equilibrium the number impinging

${ }^{6}$ Tolman, This Journal, 42, 1185 (1920).

${ }^{7}$ In the article referred to, it was shown that the entropy of a number of monatomic gases at $298^{\circ} \mathrm{K}$ and one atmosphere pressure could be calculated from the formula,

$$
S=\frac{3}{2} R \ln M+25.1 \text { calories per degree per mol. }
$$

Comparing with Equation 2, it is evident that we may write,

$$
\frac{5}{2} R \ln T-R \ln p+S_{1}=25.1
$$

Putting $R=1.985$ calories per degree, $T=298^{\circ} \mathrm{K}, p=1,013,200$ dynes per sq. $\mathrm{cm}$., and solving for $S_{1}$ we obtain the value 24.27 .

Some of the methods for deriving Equation 2 based on the quantum theory lead to a theoretical value for $S_{1}$. Nevertheless a complete agreement as to this theoretical value does not yet seem to have been achieved. For this reason it has seemed to the present writer desirable to use the experimental value. 
on the filament surface must be equal to the number leaving the surface, and this latter will be equal to the number reflected by the surface plus the number freshly emitted by the surface. If $r$ is the fraction of the impinging electrons which are reflected and $i$ is the saturation current per unit area which can be obtained when all the electrons emitted by the surface are removed as fast as formed, and we assume that the emission of electrons from the hot filament takes place in the same way under equilibrium conditions as when the saturation current is being measured, we may write as an expression for the equality of the number of electrons impinging on the surface and leaving the surface per unit time,

$$
N=r N+\frac{i}{F}
$$

where $F$ is the Faraday (i. e., the quantity of electric charge carried by one mol of electrons). Substituting the value of $N$ given by Equation 3 and solving for the pressure $p$, we obtain,

$$
p=\frac{i \sqrt{2 \pi M R T}}{F(1-r)} \text {. }
$$

In order to use this equation for the case of the electrons in equilibrium with hot filaments we shall use Langmuir's ${ }^{8}$ careful measurements of the saturation currents for tungsten, tantalum and molybdenum filaments at $2000^{\circ} \mathrm{K}$, as given in the second column of Table I. For $M$, the molecular weight of the electron we shall take $5.44 \times 10^{-4} \mathrm{~g}$., for $R, 8.315 \times$ $10^{-7}$ ergs per degree per mol, for $T, 2000^{\circ} \mathrm{K}$, and for $F, 96540$ coulombs.

As to the value for $r$ for the fraction of the electrons reflected we have very meager data. Richardson ${ }^{9}$ estimates that about $30 \%$ of the slow moving electrons present in the absence of an electric field are reflected from a brass surface. Gehrts ${ }^{10}$ found $42 \%$ reflection for aluminum, $58 \%$ for lead, $52 \%$ for cobalt, $56 \%$ for copper and $10 \%$ for lamp-black surfaces with electrons which had been emitted from a copper plate by the action of ultra-violet light and then accelerated by a drop of potential of 2 volts. When no voltage drop was applied the percentage reflection was less in those cases where measurements were actually made, but there is some uncertainty as to the exact interpretation of the results owing to the possible disturbing effect of gas molecules left in the evacuated tube.

Fortunately very exact information as to $r$ is not necessary, since it will be seen from an examination of Equations 2 and 5 that it enters into the final expression for entropy as an additive term of the form,$$
R \ln (1-r) \text {. }
$$

- Langmuir, Trans. Electrochem. Soc., 29, 125 (1916).

${ }^{9}$ Richardson, loc. cit., p. 155.

${ }^{10}$ Gehrts, Ann. Physik., 36, 995 (1911).
} 
If $r$ has the value zero this term has the value zero. If $r$ should have a value as great as 0.5 , the whole term would only have the value of 1.4 entropy units in a total which is going to be about 50 entropy units. Under the circumstances we shall neglect the reflection altogether and put $r=0$.

Substituting the above numerical values into Equation 5 we have obtained values for the pressures of the electron gas in equilibrium with the filaments, as given in the third column of Table I. It will be seen that the pressures of the electron gases in equilibrium with these filaments are so exceedingly low as to give: us confidence in our assumption that the gas laws are valid.

TABLE I.

\begin{tabular}{|c|c|c|c|}
\hline Filament. & $i_{2000^{\circ}}$. $\frac{\text { arnperes }}{\text { sq. cm. }}$ & $p \frac{\text { dynes }}{\text { sq.cm. }}$ & $S_{2000^{\circ}}$ \\
\hline Tungsten............... & 0.0042 & $1.037 \times 10^{-3}$ & 53.3 \\
\hline 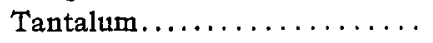 & 0.007 & $1.729 \times 10^{-3}$ & 52.3 \\
\hline Molybdenum...$\ldots \ldots \ldots \ldots$ & 0.013 & $3.210 \times 10^{-3}$ & 51.0 \\
\hline
\end{tabular}

Numerical Values of Entropy from Equation 2.--We are now ready to substitute into the theoretical Equation 2. Expressing entropy in calories per degree we shall take $R=1.985$ calories per degree, $T=$ $2000^{\circ} \mathrm{K}, M=5.44 \times 10^{-4}$ grams, and $S_{1}=24.27$. Substituting these values together with the pressures just determined into Equation 2 we have obtained the values for the entropy of the electron gases given in the fourth column of Table I.

\section{Calculation of the Entropy of Electron Gases from Heat of Vapori- zation.}

It has long been realized from the work of Richardson, that the emission of electrons from a hot filament is accompanied by an absorption of heat. This was made evident in the first place by the great temperature coefficient for the thermal emission of electrons. More recently, however, Richardson ${ }^{2}$ has actually shown and measured the cooling of a filament when a given number of electrons are allowed to escape and the inverse effect of heating when the electrons are absorbed. Fairly exact measurements of the cooling effect have more recently been made by Lester. ${ }^{11}$

If $\Delta H$ is the heat absorbed when a mol of electrons is reversibly evaporated at temperature $T$, it is evident that the increase in entropy of the system will be given by the equation,

$$
\Delta S=\frac{\Delta H}{T} .
$$

${ }^{11}$ Lester, Phil. Mag., 31, 197 (1916). 
'This increase in entropy is obviously equal to the entropy $S$ of the electron gas which has been formed in plus the change in the entropy of the metal $\Delta S_{m}$ which occurs when one mol of electrons is removed, so that we may rewrite Equation 6 in the form,

$$
S=\frac{\Delta H}{T}-\Delta S_{m} .
$$

The quantity $\Delta S_{m}$ which is the change in the entropy of a large quantity of metal when one mol of electrons is removed is presumably small, since owing to the small mass of electrons and the strong constraints under which they are held within the metal they are known from specific heat data to pick up thermal energy only very slowly as the temperature is raised above the absolute zero. For example, in the case of tungsten, the specific heat at room temperature $\left(300^{\circ} \mathrm{K}\right)$ is known to have about the theoretical value $C_{v}=6.0$, predicted on the assumption that the electrons have acquired no thermal energy. At $2000^{\circ}$ Worthing ${ }^{12}$ finds $C_{v}=7.0$. If we assumed that the partial specific heat of the electrons over the whole range from $300^{\circ} \mathrm{K}$ to $2000^{\circ} \mathrm{K}$ were the whole difference between these figures or one calorie per degree, the partial entropy acquired by all the electrons in one mol of tungsten at $2000^{\circ}$ would only be 1.9 entropy units. Under the circumstances we shall feel justified in neglecting the change in the entropy of the metals in question when the electrons are evaporated and shall write directly,

$$
S=\frac{\Delta H}{T}
$$

where $S$ is the entropy of the electron gas, the same quantity which we have already calculated from the theoretical Equation 2.

The Heat of Vaporization of Electron Gas.-In order to carry out the calculations indicated by Equation 8, we shall make use of the direct determinations of Lester ${ }^{11}$ of the heat lost by a filament when electrons are evaporated from it. These measurements were made by comparing the change in the resistance of a filament produced by the cooling effect of electron emission with the change in resistance produced by a known change in the current used for heating the filament.

Lester has calculated his results on the assumption that the electrons leaving the hot filament have the kinetic energy $2 R T$ per mol which, in accordance with Maxwell's distribution law is the average kinetic energy of the molecules of a gas which cross any plane boundary in a given time interval. Since the experimental results of Richardson ${ }^{13}$ and his coworkers have actually demonstrated that the velocities of the electrons emitted from a hot filament are distributed in accordance with the Max-

${ }^{12}$ Worthing, Phys. Rev., 12, 199 (1918).

is Richardson, op. cit., p. 139 and following. 
well law, we may accept this assumption. Lester's calculations also assume that the electrons in the metallic part of the circuit bring with them into the hot filament the energy $2 R T_{c}$ where $T_{c}$ is the temperature of the cool part of the circuit. This latter assumption is presumably not an exact approximation of the truth. Nevertheless the whole correction term, $2 R(T-T c)$, is only a few per cent. of the total heat effect so that our lack of exact information is not serious.

Lester's results may be expressed with the help of the equation,

$$
P=F \phi+2 R(T-T c)
$$

where $P$ is the actual energy input per second which Lester found was necessary in order to produce the same numerical change in filament temperature as was produced by the evaporation of one mol of electrons per second; $F$ is the Faraday or quantity of electricity carried by one mol of electrons, $\phi$ is the equivalent potential drop which the electrons have to overcome in escaping through the surface of the metal and the correction term $2 R(T-T c)$ has the significance already discussed.

For $\phi$ Lester gives the values in volts shown in the second column of Table II. If we multiply $\phi$ by $F=96540$ coulombs, we find the energy input in joules necessary to take one mol of electrons across the boundary of the metal, under the conditions of the experiment and shall assume this same energy input necessary for a reversible evaporation. In order to get the reversible heat of vaporization we must add to the energy input the quantity $R T$ which is the external work that would have to be done against a movable piston if one mol of electrons were reversibly evaporated We obtain for the heat of vaporization, ${ }^{14}$

Taking $T=2000^{\circ} \mathrm{K}$ and expressing $\Delta H$ in calories, the values obtained are given in the third column of Table II. Substituting in Equation 8

14 It should be noted that this expression for $\Delta H$ is based on Lester's assumption that the electrons in a metal at temperature $I$ have the same mean kinetic energy as the molecules of a perfect gas at the same temperature, which does not agree with the low specific heat of metals already referred to above in connection with our estimate of $\Delta S_{m}$.

If we assumed that the electrons in the metal had no kinetic energy, we could then get $\Delta H$ as follows. Take Lester's value of $P$, substract $2 R T$ which is the kinetic energy of the escaping electrons when the saturation current is being measured, add ${ }_{3}^{3} / 2 R T$ which would be the kinetic energy of the electrons in the space surrounding the filament of electrons under equilibrium conditions and add $R T$ for the external work. We should obtain

or substituting Lester's expression for $P$

$$
\Delta H=P-2 R T+3 / 2 R T+R T
$$

$$
\Delta H=F \phi+5 / 2 R T-2 R T_{c}
$$

Since $T_{c}$ was actually a little less than $T$, it will be seen that expression used above for $\Delta H$ was not greatly different from that which would be calculated by this other method.

$$
\Delta H=F \phi+R T .
$$


we obtain the values given in the fourth column of Table II for the entropy of one mol of electron gas in equilibrium with tungsten, tantalum and molybdenum filaments at $2000^{\circ} \mathrm{K}$. For comparison the values calculated by the theoretical Equation 2 are given in the last column of the table.

TABLE II.

\begin{tabular}{|c|c|c|c|c|}
\hline Filament. & $\Phi$ volts. & $\Delta H$ & $S=\frac{\Delta H}{T}$ & $S$ by Equation 2 \\
\hline Tungsten. & 4.478 & 107270 & 53.6 & 53.3 \\
\hline Tantalum. & 4.511 & 107970 & 54.0 & 52.3 \\
\hline Molybdenum. . & 4.588 & 109770 & 54.9 & 51.0 \\
\hline
\end{tabular}

\section{Discussion.}

The Data Selected.-The three filaments considered, namely, tungsten, tantalum and molybdenum are the only ones for which both Langmuir has given values of the saturation current and Lester values of the heat of vaporization. Values for the heat of vaporization $\Delta H$ might also have been obtained by making use of the Clausius vapor-pressure equation,

$$
\frac{d \ln p}{d T}=\frac{\Delta H}{R T^{2}}
$$

by substituting the value for pressure $p$ in terms of saturation current $i$ given by Equation 5 ,

$$
p=\frac{i \sqrt{2 \pi M R T}}{F(1-r)} .
$$

This would permit us to extend our considerations to any filament for which we have accurate values of the saturation current and the rate of change of saturation current with the temperatures. The comparisons seemed more conclusive, however, if we restricted ourselves to the case, of heats of vaporization which have been directly determined. Moreover it should be noted that in using the indirect method of determining heats of vaporization, we should have to introduce the additional assumption that $\mathrm{d} r / \mathrm{d} T$ as well as $r$ is a negligible quantity. Furthermore, the data on the rate of change of saturation current with temperature are usually expressed with the help of Richardson's original formula $i=a T^{1 / 2} \epsilon^{b / t}$ where $a$ and $b$ are constants, and since this formula has no completely satisfactory theoretical justification, we might be led into error if we made use of it over a wider range of temperature than that in which it is empirically reasonably valid. ${ }^{15}$

${ }^{15}$ Making use of Langmuir's data (loc. cit.,) for the rate of change of saturation current with the temperature, and calculating $\frac{\Delta H}{T}$ by the method described above, the following results have been obtained for the entropy of electron gas in equilibrium with 
The Agreement.-The agreement between the values for the entropy of electron gas as given by the theoretical Equation 2 and as calculated from the known heats of vaporization agree very well as shown in the last two columns of Table II. It is believed that the results may be taken as an adequate proof that the entropy of a dilute electron gas may be calculated from the same theoretical equation as has been found to apply to other monatomic gases. Of course, an exact agreement between the two methods of calculation is hardly to be expected, first because of experimental error in the data employed and secondly because of a number of assumptions which had to be introduced in the course of the calculations in order to arrive at any conclusions at all.

The Assumptions.-A recapitulation and discussion of these assumptions will not be out of place.

(a) In order to obtain Equation 5 for the relation between saturation current and the pressure of the electron gas in equilibrium with a hot filament, it was assumed that the same number of electrons will be given off per second by a hot filament, when electron gas is in equilibrium with the filament and when the saturation current is being determined. A partial justification for this assumption is provided by the fact that the current is found to be independent of the anode potential as soon as a high enough voltage drop has been applied to overcome the backing up effect of the space charge. With the Coolidge X-ray tube this has been shown to be true even at voltages above 100,000 volts. ${ }^{16}$ Under the circumstances, we may conclude that the electron emission will not be decreased by the small-potential drop in the opposite direction present under equilibrium conditions.

To complete the justification for our assumption that the electron emission is the same under equilibrium conditions when the saturation current is being determined, we also need assurance that under equilibrium conditions no secondary emission is produced by electrons which bombard the emitting walls, since such a bombardment is not present when the saturation current is being determined. This seems to be justified in view of the work of Gehrts, already referred to, which shows that a potential drop of about 11 volts is necessary to produce secondary emission in the case of surfaces of aluminum, lead, cobalt and copper, and this corre-

hot filaments at $2000^{\circ} \mathrm{K}$. The values given in the last column are those obtained from the theoretical Equation 2 by the method already described.

$\begin{array}{lrc}\text { Filament. } & \mathrm{S}=\frac{\Delta H}{T} . & S \text { theoretical } \\ \text { Tungsten ....... } & 54.1 & 53.3 \\ \text { Tantalum........ } & 51.6 & 52.3 \\ \text { Molybdenum..... } & 51.6 & 51.0 \\ \text { Thorium. . . . . . } & 40.7 & 31.1 \\ \text { Phys. Rev., 2, } 409 \text { (1913), } & \end{array}$

16 Coolidge, Phys. Rev., 2, 409 (1913). 
sponds to a much higher kinetic energy than that of the average molecule at $2000^{\circ}$.

(b) In making actual calculations of the pressure with the help of Equation 5 it was assumed that the reflecting power of the hot metals in question is zero. This is probably not strictly true, in view of the work of Gehrts ${ }^{10}$ on the reflecting power of a number of cold metallic surfaces. Nevertheless the effect of possible condensed layers of gas might increase the reflecting power of cold surfaces such as those with which he worked. Moreover, as already pointed out, a reflecting power anywhere between 0 and $50 \%$ would have but little effect on the final value of the entropy.

(c) In calculating the entropy of electron gas from its heat of vaporization, we have assumed that the values of Lester for the heat of vaporization when the vaporized electrons are being removed as fast as emitted is the same as the heat of vaporization under equilibrium conditions. This assumption is obviously closely connected with assumption (a) that the emission of electrons takes place to the same extent under equilibrium conditions and when the saturation current is being determined. Since assumption (a) occurred in the first method of calculating entropy and assumption $(c)$ in the second method of calculation, the errors introduced, if any, may have cancelled out.

(d) In calculating the entropy of electron gas from its heat of vaporization, we have also neglected the change in the entropy of a metal produced by a change in its electric charge. This assumption is probably not strictly correct. The electrons in a metal, however, are held by strong constraints and have a small mass and hence pick up appreciable amounts of thermal energy only at high temperatures where, in accordance with the fundamental equation $\mathrm{d} S=\mathrm{d} Q / T$ the rate of increase in entropy compared with the rate of increase in heat content is small.

Conclusion.--It is believed that this article establishes with reasonable certainty the possibility of calculating the entropy of electron gas from the theoretical equation which has been found to apply to other monatomic gases, namely,

$$
S=5 / 2 R \ln T-R \ln p+3 / 2 R \ln M+S_{1}
$$

where $S_{1}$ has the same value for all monatomic gases.

WASHINGTON, D. C. 